\title{
The experimental quest for in-medium effects
}

\author{
Romain Holzmann*广 \\ GSI Helmholtzzentrum für Schwerionenforschung mbH \\ 64291 Darmstadt \\ Germany \\ E-mail: r.holzmann@gsi.de
}

The QCD vacuum is characterized by finite expectation values of various quark and gluon operators. Most notable is the $\bar{q} q$ condensate which signals the spontaneous breaking of chiral symmetry. In a strongly interacting medium the chiral condensate is expected to be modified, and that already by about $30 \%$ at nuclear saturation density and zero temperature. Supposing, as suggested e.g. by QCD sum rules, that vector meson properties are indeed related to the QCD condensates, changes of the latter should be evidenced in particular in the decays $\rho, \omega, \phi \rightarrow e^{+} e^{-}$. This contribution briefly reviews the status of the experimental investigation of in-medium effects on vector mesons, illustrated with a few results obtained with the HADES dielectron spectrometer at GSI.

PACS: $13.20 . \mathrm{Gd}, 13.60 .-\mathrm{r}, 25.40 .-\mathrm{h}, 25.75 .-\mathrm{q}, 25.75 . \mathrm{Cj}$

Sixth International Conference on Quarks and Nuclear Physics

April 16-20, 2012

Ecole Polytechnique, Palaiseau, Paris

\footnotetext{
* Speaker.

${ }^{\dagger}$ Member of the HADES Collaboration
} 


\section{Expectations from theory}

While the mass of the proton is of order $1 \mathrm{GeV} / \mathrm{c}^{2}$, the Higgs generated current masses of its three valence quarks add up to only about $20 \mathrm{MeV} / \mathrm{c}^{2}$. Obviously other mass-generating mechanisms must be at play! In fact, besides the explicit breaking of the chiral symmetry of the QCD lagrangian due to the small, but non-vanishing current quark masses, it is now understood that the large masses of hadrons are related to an additional spontaneous breakdown of this symmetry. The vacuum state of QCD is highly non-trivial: a non-zero scalar quark condensate is built up with the spontaneous breaking of chiral symmetry. The interaction of almost massless current quarks with the scalar and higher-order quark and gluon condensates do in this picture lead to heavy constituent quarks which then give rise to the observed large hadron masses. In nuclear matter the quark condensates are thought to be quenched and, consequently, the chiral symmetry would be partially restored. The study of hadron properties - effective masses, decay widths, electromagnetic form factors - inside the nuclear medium at any density and excitation energy is hence of fundamental interest. The partial restoration of the QCD chiral symmetry is expected to lead to a mass change of, in particular, vector and axial-vector mesons with finite temperature and density of the surrounding nuclear medium. More specifically, QCD sum rules [1-3] and various hadronic models [4-10] predict significant changes in mass and resonance width of the light vector mesons, i.e. $\rho, \omega$ and $\phi$, when embedded into nuclear matter. Models based on a mean-field approach, the bag model, or constituent quark models [11-13] predict such changes too. For a recent review of this research field see $[14,15]$.

These theoretical concepts are all very attractive, but experimental verification is required. The challenge is to measure the properties of hadrons in hot and/or dense hadronic matter. To probe in-medium effects, vector mesons are best suited because the very short-lived $\rho$ mesons $\left(\tau_{v a c}=\right.$ $1.3 \mathrm{fm} / \mathrm{c}$ ) decay mainly inside the nucleus or a reaction zone of comparable extension, and even the longer-lived $\omega$ mesons $\left(\tau_{v a c}=23 \mathrm{fm} / \mathrm{c}\right)$ and $\phi$ mesons $\left(\tau_{v a c}=44 \mathrm{fm} / \mathrm{c}\right)$ still do so to a substantial fraction [16, 17]. A variety of reactions allow to scan the parameter space of hadron density vs. temperature: vector mesons can be produced in photon-, hadron- and heavy-ion induced reactions, and a complete measurement of their decay products gives in principle access to the full range of the in-medium effects mentioned above. As photons and leptons do not undergo strong final-state interactions, they are ideally suited for probing particle decays inside the nuclear medium. Hence, direct photon and lepton pair spectroscopy is the most promising tool to investigate medium effects and, in addition, may help to pin down the gross thermodynamic properties (chemical potentials, temperature) of hot nuclear matter. On the other hand, a full description of in-medium hadron properties can only be reached with a thorough understanding of the vacuum properties of all involved states, i.e. the vector mesons themselves as well as the baryon resonances to which they couple. This means that dilepton studies have to be pursued systematically, not only in heavy-ion reactions, but as well in proton- and pion-induced reactions, i.e. in AA, pA, $\gamma \mathrm{A}, \mathrm{pp}, \mathrm{pn}$, and $\gamma \mathrm{p}$ reactions. The HADES detector at GSI [18] is particularly well suited to support a systematic experimental program of dielectron spectroscopy in a variety of reactions accessible with beams from SIS18 in the few-GeV bombarding energy regime. 


\section{The experimental approach}

Experimentally, in-medium properties are studied either in heavy-ion collisions (HIC), probing hot and dense hadronic matter, or in proton- and photon-induced reactions on nuclei, probing cold nuclear matter. Whereas medium modifications are expected to be stronger in HIC, their measurable observables represent an average over the complete space-time evolution of the collision. In contrast, using reactions with elementary projectiles, the investigated system does not cross a steep density and temperature profile, and observed quantities correspond to better defined conditions. In both cases, lepton pair $\left(\mathrm{e}^{+} \mathrm{e}^{-}\right.$or $\left.\mu^{+} \mu^{-}\right)$decays of vector mesons are ideal probes since electrons and muons are not affected by strong final-state interactions.

The dominant decay channels of vector mesons are hadronic, but there is a small branching of the order of $10^{-5}$ for decays into a $\mathrm{e}^{+} \mathrm{e}^{-}$or $\mu^{+} \mu^{-}$pair. First dilepton invariant-mass spectra were reported from proton- and pion-induced reactions at typical center-of-mass energies of $6 \mathrm{GeV}<$ $\sqrt{s}<30 \mathrm{GeV}$. In $\mathrm{pN}$ reactions investigated at the CERN SPS, the measured yield in the dielectron invariant-mass distribution could be explained by decays of free (i.e. in-vacuum) hadrons. At low invariant masses $\left(\mathrm{M}<0.6 \mathrm{GeV} / \mathrm{c}^{2}\right)$ the spectrum is mostly populated by the Dalitz decays of $\pi^{0}, \eta$ and $\omega$ mesons. At higher masses, however, the contributions from pair decays of the $\rho^{0}, \omega$ and $\phi$ mesons dominate. On the other hand, in this mass region, dileptons originating from charm production and/or from direct quark-antiquark annihilation (Drell-Yan process) are of minor importance. Respective spectra were taken at the SPS by the HELIOS (p + Be [19]) and later by the CERES ( $\mathrm{p}+\mathrm{Be}$ and $\mathrm{p}+\mathrm{Au}[20])$ experiments, all at proton energies of $450 \mathrm{GeV}$. Similar observations were made by the NA38 collaboration for dimuons in the system $\mathrm{p}+\mathrm{U}$ and $\mathrm{p}+\mathrm{Cu}$ [21]. Likewise, at lower bombarding energies, in proton-induced reactions $(\mathrm{p}+\mathrm{p}$ and $\mathrm{p}+\mathrm{d})$ measured by the DLS collaboration at the Bevalac [22] and the HADES collaboration at GSI [23] for beams energies between 1 and $5 \mathrm{GeV}$, the dielectron yield can quantitatively be understood by a superposition of freely decaying hadrons [24-27].

The experimental situation, however, changes drastically when going from proton to heavy-ion beams. Independent of the bombarding energy, in all dilepton spectra taken, an enhancement compared to the superposition of free hadronic decays becomes visible in the mass region of $0.2 \mathrm{GeV} / \mathrm{c}^{2}$ $<M_{i n v}<0.6 \mathrm{GeV} / \mathrm{c}^{2}$. This observation was first made by the CERES and HELIOS experiments for dielectrons in the system $\mathrm{S}+\mathrm{Au}$ [28] and for dimuons in the system $\mathrm{S}+\mathrm{W}$ [29], respectively. Later on, a similar behavior was found in $\mathrm{Pb}$-induced collisions at $159 \mathrm{~A} \mathrm{GeV} \mathrm{[30]} \mathrm{and,} \mathrm{more} \mathrm{re-}$ cently, at $40 \mathrm{~A} \mathrm{GeV}$ [31]. It was observed that the dielectron yield increases more strongly with charged-particle multiplicity as compared to the proton reaction, a clear indication for medium effects. Analogously, at Bevalac energies, the DLS collaboration has observed a significant enhancement in the systems $\mathrm{C}+\mathrm{C}$ and $\mathrm{Ca}+\mathrm{Ca}$ at a beam energy of $1.04 \mathrm{~A} \mathrm{GeV} \mathrm{[32].} \mathrm{The} \mathrm{latter} \mathrm{data}$ are of particular interest, as early calculations based on BUU transport theory assuming a cocktail of decaying baryon resonances and mesons, as well as pion annihilation [33] could not reproduce the measured enhancement, even if medium effects in the $\rho$ propagation were taken into account. On the other hand, at these energies, contributions from the $\Delta$ Dalitz decay, as well as from $\mathrm{pN}$ and pn bremsstrahlung are relevant sources, since the fireball is still dominated by baryons. Yet various other transport calculations failed as well [24, 34]. Furthermore, the TAPS measurements of $\eta$ production in the two-photon decay channel [35] have given direct experimental evidence that the 
$\eta$ Dalitz branch alone can not be at the origin of the observed enhancement. This state of affairs in the few-GeV bombarding energy range became known as the "DLS puzzle" and still awaits a fully satisfying solution (see next section). On the other hand, the SPS data are reproduced quite well by hadronic many-body theories (HMBT) invoking in-medium spectral functions of vector mesons, in particular the $\rho$ (see e.g. [8,36-38]). More recently the HADES experiment has started a series of comprehensive dielectron measurements in $\mathrm{C}+\mathrm{C}, \mathrm{Ar}+\mathrm{KCl}$, and $\mathrm{Au}+\mathrm{Au}$ reactions at beam energies of a few $\mathrm{GeV}$; they are discussed in the next section.

Reactions with beams of photons and protons allow to investigate dilepton production at low temperature and normal nuclear matter density. Various such measurements have been executed by the CBELSA/TAPS experiment at ELSA [39], the CLAS experiment at JLab [40], and the E325 experiment at KEK [41, 42]. Specific changes in the reconstructed spectral shape of rho, omega and phi mesons have been reported, but no consistent picture has yet emerged from this body of data. Very recently, a refined analysis of the NA60 dimuon [43] and the CERES dielectron [44] data allowed to extract, by subtraction of the long-lived part of the cocktail of dilepton sources, the in-medium spectral shape of the $\rho$ meson. Both data sets are consistent with a strong broadening of the rho, rather than a mass shift. These results, when compared to calculations based on hadronic multi-body theories, allow to gain direct information on the medium-modified rho spectral function $[37,38]$. A firm conclusion on whether chiral symmetry is actually restored in dense and hot hadronic matter can, however, not easily been drawn from such data alone as it requires to disentangle the QCD-driven from the hadronic effects $[14,15]$. It has furthermore been argued $[15,45]$ that, because of strong collisional broadening, the in-medium widths of the longer-lived $\omega$ and $\phi$ mesons are very difficult to observe directly. Medium modifications should rather be accessible by measuring the target-size dependence of their production cross sections. Such data, coded in terms of a so-called transparency ratio $T_{A}$, have lately been provided by the CBELSA/TAPS [39, 46], the CLAS [47], the LEPS [48], and the ANKE [49] experiments ${ }^{1}$. Their theoretical interpretations $[45,50]$ points indeed to a very strong in-medium broadening of the $\omega$ and $\phi$ vector mesons. In this context, HADES has obtained particularly interesting results for low pair momenta by comparing dielectron production in $3.5 \mathrm{GeV} \mathrm{p}+\mathrm{p}$ and $\mathrm{p}+\mathrm{Nb}$ reactions.

\section{The HADES experiment at GSI}

With the High Acceptance DiElectron Spectrometer HADES electron-positron pair spectroscopy is available at GSI for studies in nucleus-nucleus reactions in the 1-2 AGeV bombarding energy regime, as well as in elementary reactions with proton, deuteron, and also secondary pion beams. This energy range, together with the large choice of collision systems, allows to access the region in the nuclear matter phase diagram ranging from ground state density $n_{0}$ up to about $3 n_{0}$, while spanning temperatures from zero up to about $0.1 \mathrm{GeV}$. In addition, at these bombarding energies, the produced fireball is still baryon dominated. Therefore, a major part of the HADES physics program consists in a systematic study of $\mathrm{e}^{+} \mathrm{e}^{-}$pair production in heavy-ion and proton-induced collisions, searching in particular for precursor effects of chiral symmetry restoration. HADES is a second-generation experiment for high-resolution electron-pair spectroscopy (for details see

\footnotetext{
${ }^{1}$ Note that the CBELSA/TAPS, LEPS and ANKE experiments reconstruct the vector meson from a hadronic decay channel, $\omega \rightarrow \pi^{0} \gamma$ and $\phi \rightarrow \mathrm{K}^{+} \mathrm{K}^{-}$, respectively.
} 
$[18,51]$ and http://www-hades.gsi.de). The key features of the HADES spectrometer shown in Fig. 1 are: (a) an excellent lepton/hadron discrimination, (b) a resolution for invariant-mass reconstruction approaching the natural width of the $\omega$ meson $\left(\sigma_{M} / M \simeq 2 \%\right)$, (c) a signal-to-background ratio significantly larger than unity for invariant masses up to $M \approx 1 \mathrm{GeV} / \mathrm{c}^{2}$, (d) a large geometrical acceptance in combination with high count rate capability, and (e) a high granularity to cope with heavy collision systems up to $\mathrm{Au}+\mathrm{Au}$. These combined features result in a large increase in sensitivity over the former DLS setup [22, 32].

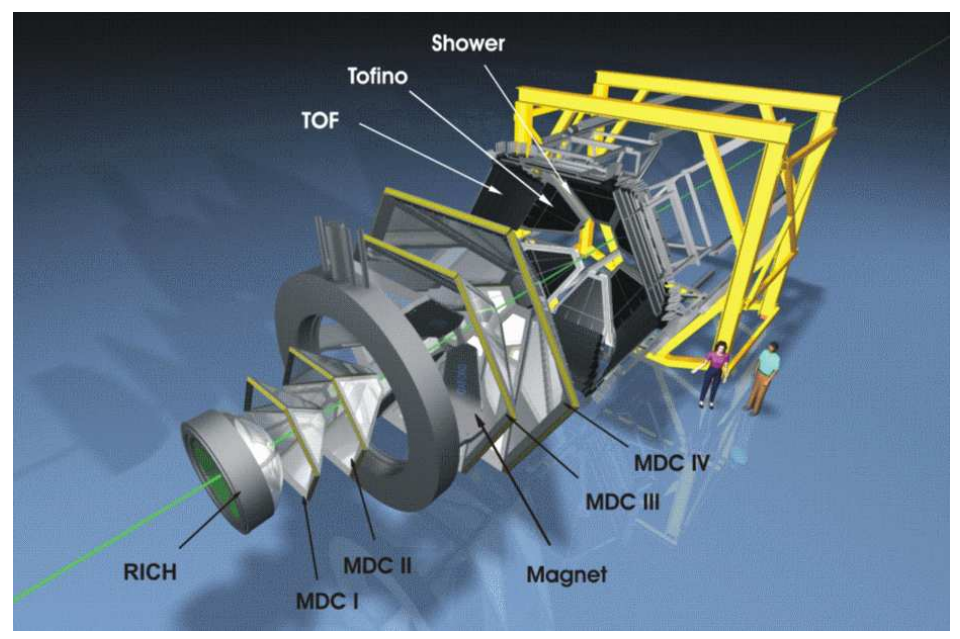

Figure 1: Exploded view of the HADES detector at GSI [18]. In 2011 the Tofino time-of-flight scintillators were replaced by fast, highly-segmented RPC modules for vastly improved timing and granularity.

In a first phase, the low granularity of the inner part of the TOF wall prohibited the use of the detector in a high-multiplicity environment. Therefore, the measurement program of HADES started out with its focus set on light collision systems: $\mathrm{p}+\mathrm{p}, \mathrm{d}+\mathrm{p}, \mathrm{C}+\mathrm{C}, \mathrm{Ar}+\mathrm{KCl}$, and p+Nb. Finally, in 2011, HADES was upgraded with the installation of RPC timing detectors, the addition of a forward plastic hodoscope for event plane and centrality characterization, and new read-out electronics boosting the performance of the DAQ system. With this configuration, data on dilepton and strangeness production in the heavy reaction system Au+Au could also be taken. Only a small selection of results can be shown below, but a detailed discussion can be found in the listed references.

In one of its first experiments, HADES has measured dielectron production in $\mathrm{p}+\mathrm{p}$ and quasifree $n+p$ collisions at $1.25 \mathrm{GeV}$ [23]. A very strong isospin dependence of the dielectron production has been found. As shown in Fig. 2, the puzzling dielectron excess in the intermediate mass range of $M_{e+e-}=0.15-0.5 \mathrm{GeV} / \mathrm{c}^{2}$ observed in $\mathrm{C}+\mathrm{C}$ collisions at $1 \mathrm{~A}$ and $2 \mathrm{~A} \mathrm{GeV}$ [52] can be described by a superposition of elementary $\mathrm{p}+\mathrm{p}$ and $\mathrm{n}+\mathrm{p}$ collisions. Although a sound theoretical description of the relevant sources is still lacking, the data indicate that this excess can be traced back essentially to effects present already in $\mathrm{n}+\mathrm{p}$ collisions. The results on $\mathrm{e}^{+} \mathrm{e}^{-}$production obtained with HADES in the medium-heavy $\mathrm{Ar}+\mathrm{KCl}$ system at $1.76 \mathrm{~A} \mathrm{GeV}$ [53] show an intermediate-mass pair excess over long-lived sources a factor $\approx 3$ stronger than the one observed in elementary nucleon-nucleon reactions and $\approx 2$ stronger than in $\mathrm{C}+\mathrm{C}$ reactions (see Fig. 3). One can indeed argue that this behavior signals the onset of the influence of the nuclear medium on dilepton production. It is expected that this enhancement will be much larger even in central $\mathrm{Au}+\mathrm{Au}$ collisions. These data, 
still in the process of being analyzed, will definitely allow to characterize dilepton emission from the hot and dense hadronic medium produced in few-GeV heavy-ion collisions.

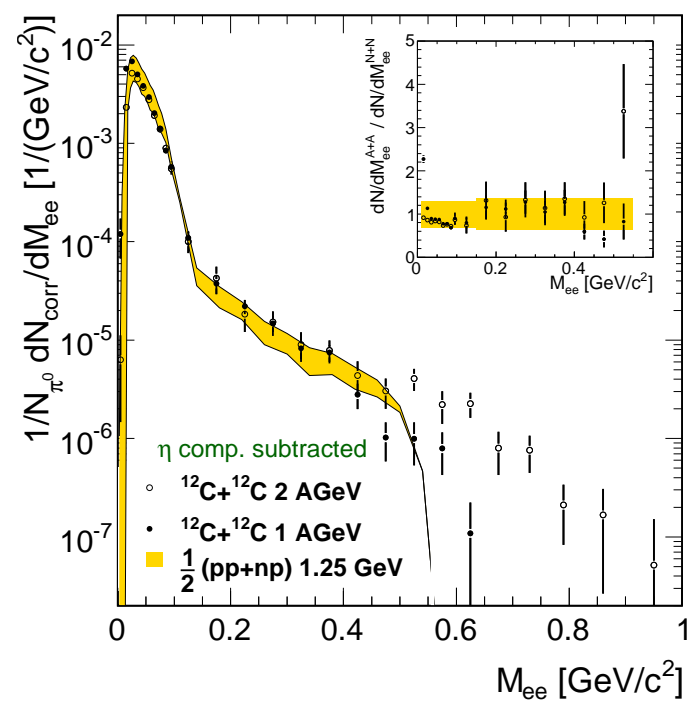

Figure 2: Comparison of $\mathrm{e}^{+} \mathrm{e}^{-}$mass distributions obtained with HADES in $1 \mathrm{~A} \mathrm{GeV}$ and $2 \mathrm{~A} \mathrm{GeV}$ $\mathrm{C}+\mathrm{C}$, and the average from $1.25 \mathrm{GeV} \mathrm{p}+\mathrm{p}$ and $\mathrm{n}+\mathrm{p}$ collisions Contributions from $\eta$ Dalitz decay have been subtracted (see [23] for details).

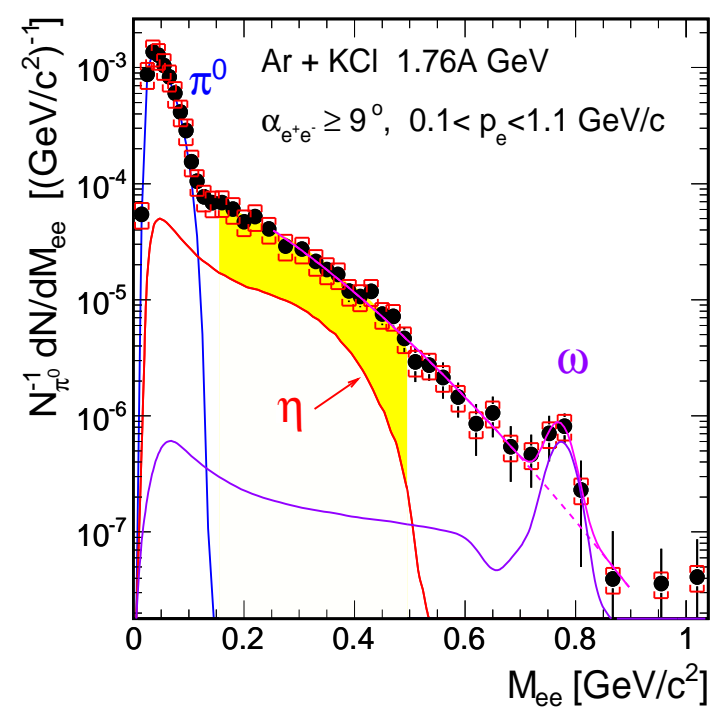

Figure 3: Dielectron mass distributions measured with HADES in $1.76 \mathrm{~A} \mathrm{GeV} \mathrm{Ar}+\mathrm{KCl}$ collisions, compared with a cocktail of long-lived sources (from [53]). The yellow band depicts the excess over this cocktail

Likewise, in $3.5 \mathrm{GeV}$ p+Nb collisions, a strong dielectron excess yield with respect to the $\mathrm{p}+\mathrm{p}$ reaction at this beam energy is seen, especially for pairs with low momenta, that is, for slow pairs [54]. HADES has indeed obtained the first high statistics measurement of dielectrons radiated from cold nuclear matter in a kinematic regime where strong medium effects are expected. The difference in the spectral shape shown in Fig. 4 in the mass region dominated by vector meson decays can be attributed to a concurrent decrease of $\omega$ yield and enhancement of $\rho$-like contributions, the latter most likely due to secondary reactions. The importance of secondary processes is signaled in both, the enhancement of low-momentum pairs over the whole mass range and the shift to target rapidity in $\mathrm{p}+\mathrm{Nb}$ compared to $\mathrm{p}+\mathrm{p}$ reactions. The lack of yield in $\mathrm{p}+\mathrm{p}$ reactions as compared to a PYTHIA calculation, as well as the observed enhancement in $\mathrm{p}+\mathrm{Nb}$ reactions support an interpretation invoking a strong coupling of the $\rho$ meson to baryonic resonances [27]. Such couplings enter in $\rho$ meson production and in-medium propagation. The observed suppression of $\omega$ emission from $\mathrm{p}+\mathrm{Nb}$ can be explained by strong collisional broadening, as proposed already in the interpretation of the data on vector meson photoproduction $[45,50]$.

\section{Outlook}

Evidently, further high-resolution experiments (CLAS, HADES, JPARC, MAMI) are needed to obtain a comprehensive and consistent picture of in-medium properties of the light vector mesons. For the next few years, HADES will hence continue its experimental program on dilepton pro- 

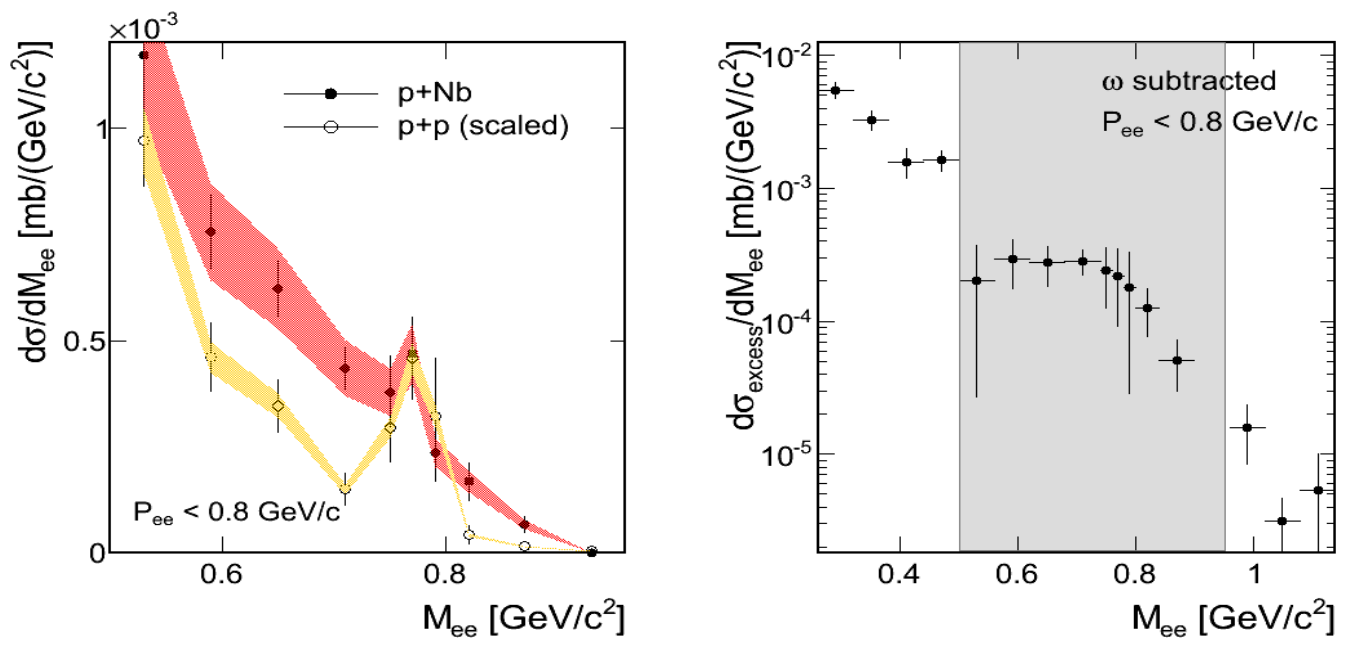

Figure 4: Left: Comparison of the invariant mass spectra of $\mathrm{e}^{+} \mathrm{e}^{-}$pairs with $P_{e e}<0.8 \mathrm{GeV} / \mathrm{c}$ from $\mathrm{p}+\mathrm{p}$ and $\mathrm{p}+\mathrm{Nb}$ reactions, respectively. The colored bands represent the systematic uncertainties due to the normalization. Right: Excess yield in $\mathrm{p}+\mathrm{Nb}$ after subtraction of the scaled $\mathrm{p}+\mathrm{p}$ reference data; the $\omega$ contribution is subtracted in both samples. The greyed region corresponds to the mass range plotted in the left picture. (Both figures are taken from [54]).

duction in heavy-ion reactions, complemented also with studies of elementary processes in pioninduced reactions using the secondary pion beams available from SIS18. The long-term perspective, beyond round about 2017, is to integrate the HADES detector into the FAIR accelerator complex, now in construction at GSI. Making use of the future SIS100 beams, with energies of up to $8 \mathrm{~A} \mathrm{GeV}$, will allow it to bridge the gap between the present SIS energies and those to be used by the future Compressed Baryonic Matter experiment, CBM at FAIR [55]. Furthermore, antiproton beams provided by the high energy storage ring (HESR) at FAIR will open up the possibility to extend the study of in-medium properties of hadrons to the charm sector.

\section{Acknowledgments}

Here I take the opportunity to thank all of my colleagues from the HADES experiment for many years of fruitful collaborative work and numerous enlightening discussions.

\section{References}

[1] T. Hatsuda and S.H. Lee, Phys. Rev. C 46, R34 (1992).

[2] Y. Koike and A. Hayashigaki, Prog. Theor. Phys. 98, 631 (1997).

[3] S. Leupold and U. Mosel, Phys. Rev. C 58, 2939 (1998).

[4] M. Herrmann, B. Friman, and W. Nörenberg, Nucl. Phys. A 560, 411 (1993).

[5] F. Klingl and W.Weise, Nucl. Phys. A 606, 329 (1996).

[6] R. Rapp, G. Chanfray, and J. Wambach, Nucl. Phys. A 617, 472 (1997).

[7] F. Klingl, T. Waas, and W. Weise, Phys. Lett. B 431, 254 (1998).

[8] R. Rapp and J. Wambach, Adv. Nucl. Phys. 25, 1 (2000).

[9] M. Post, S. Leupold, and U. Mosel, Nucl. Phys. A 741, 81 (2004).

[10] P. Mühlich et al., Nucl. Phys. A 780, 187 (2006). 
[11] G. E. Brown and M. Rho, Phys. Rev. Lett. 66, 2720 (1991); Phys. Rep. 363, 85 (2002).

[12] K. Saito, K. Tsushima, and A.W. Thomas, Nucl. Phys. A 609, 339 (1996).

[13] C. M. Shakin and W.-D. Sun, Phys. Rev. C 49, 1185 (1994).

[14] R. S. Hayano and T. Hatsuda, Rev. Mod. Phys. 82, 2949 (2010).

[15] S. Leupold, V. Metag and U. Mosel, Int. J. Mod. Phys. E19, 147 (2010).

[16] W. Schön et al. (HADES Collaboration), Acta Phys. Pol. B 27, 2959 (1996).

[17] J. G. Messchendorp et al. (CBELSA/TAPS Collaboration), Eur. Phys. J. A 11, 95 (2001).

[18] G. Agakishiev et al. (HADES Collaboration), Eur. Phys. J. A 41, 243 (2009).

[19] T. Akesson et al. (HELIOS Collaboration), Z. Phys. C 48, 47 (1995).

[20] G. Agakishiev et al. (CERES Collaboration), Eur. Phys. C 4, 231 (1998).

[21] M. C. Abreu et al. (NA38 Collaboration), Nucl. Phys. A 590, 117c (1995).

[22] W. K. Wilson et al. (DLS Collaboration), Phys. Rev. C 57, 1865 (1998).

[23] G. Agakishiev et al. (HADES Collaboration), Phys. Lett. B 690, 118 (2010);

Eur. Phys. J. A 48, 64 (2012); Phys. Rev. C 85, 054005 (2012).

[24] C. Ernst et al., Phys. Rev. C 58, 447 (1998).

[25] A. Fässler et al., J. Phys. G 29, 603 (2003).

[26] U. Mosel and R. Shyam, Phys. Rev. C 67, 065202 (2003).

[27] J. Weil, H. van Hees, and U. Mosel, arXiv:1203.3557 [nucl-th].

[28] G. Agakishiev et al. (CERES Collaboration), Phys. Rev. Lett. 75, 1272 (1995).

[29] M. Masera et al. (HELIOS Collaboration), Nucl. Phys. A 590, 93c (1995).

[30] G. Agakishiev et al. (CERES Collaboration), Phys. Lett. B 422, 405 (1998).

[31] D. Adamova et al. (CERES Collaboration), Phys. Rev. Lett. 91, 042301 (2003).

[32] R. J. Porter et al. (DLS Collaboration), Phys. Rev. Lett. 79, 1229 (1997).

[33] E. L. Bratkovskaya et al., Nucl. Phys. A 634, 168 (1998).

[34] K. Shekhter et al., Phys. Rev. C 68, 014904 (2003).

[35] R. Holzmann et al. (TAPS Collaboration), Phys. Rev. C 56, R2920 (1997).

[36] R. Rapp and J. Wambach, Nucl. Phys. A 661, 33c (1999).

[37] H. van Hees and R. Rapp, Phys. Rev. Lett. 97, 102301 (2006).

[38] J. Ruppert,et al., Phys. Rev. Lett. 100, 162301 (2008).

[39] M. Kotulla et al. (CBELSA/TAPS Collaboration), Phys. Rev. Lett. 100, 192302 (2008).

[40] R. Nasseripour et al. (CLAS Collaboration) Phys. Rev. Lett. 99, 262302 (2007).

[41] M. Naruki et al. (KEK-PS E325 Collaboration), Phys. Rev. Lett. 96, 092301 (2006).

[42] R. Muto et al., (KEK-PS E325 Collaboration) Phys. Rev. Lett. 98, 042501 (2007).

[43] R. Arnaldi et al. (NA60 Collaboration), Phys. Rev. Lett. 96, 162302 (2006).

[44] D. Adamova et al. (CERES Collaboration), Phys. Lett. B 666, 425 (2008).

[45] P. Mühlich and U. Mosel, Nucl. Phys. A 773, 156 (2006).

[46] M. Nanova et al. (CBELSA/TAPS Collaboration), Phys. Rev. C 82, 035209 (2010);

Eur. Phys. J. A 47, 16 (2011).

[47] M. H. Wood et al. (CLAS Collaboration), Phys. Rev. Lett. 105, 112301 (2010).

[48] T. Ishikawa et al. (LEPS Collaboration), Phys. Lett. B 608, 215 (2005).

[49] A. Polyanskiy et al. (ANKE Collaboration), Phys. Lett. B 695, 74 (2011).

[50] M. Kaskulov, E. Hernandez, and E. Oset, Eur. Phys. J. A 31, 245 (2007).

[51] R. Schicker et al., Nucl. Instr. Meth. A 380, 586 (1996).

[52] G. Agakishiev et al. (HADES Collaboration), Phys. Rev. Lett. 98, 052302 (2007);

Phys. Lett. B 663, 43 (2008).

[53] G. Agakishiev et al. (HADES Collaboration), Phys. Rev. C 84, 014902 (2011).

[54] G. Agakishiev et al. (HADES Collaboration), arXiv:1205.1918v1 [nucl-ex].

[55] B. Friman et al., Lecture Notes on Physics 814, 11 (2011). 